\title{
Tunable waveguide bends with graphene-based anisotropic metamaterials
}

Zhao-xian Chen ${ }^{1,2}$, Ze-guo Chen ${ }^{1}$, Yang Ming ${ }^{2}$ and Ying $\mathrm{Wu}^{1} *$, Yan-qing $\mathrm{Lu}^{2} *$

${ }^{1}$ Division of Computer, Electrical and Mathematical Sciences and Engineering, King Abdullah University of Science and Technology (KAUST), Thuwal 23955-6900, Saudi Arabia

${ }^{2}$ National Laboratory of Solid State Microstructures and College of Engineering and Applied Sciences, Nanjing University, Nanjing 210093, P. R. China

E-mail: ying.wu@kaust.edu.sa,yqlu@nju.edu.cn

We design tunable waveguide bends filled with graphene-based anisotropic metamaterials to achieve nearly perfect bending effect. The anisotropic properties of the metamaterials can be described by effective medium theory. The nearly perfect bending effect is demonstrated by finite element simulations of various structures with different bending curvatures and shapes. This effect is attributed to zero effective permittivity along the direction of propagation and matched effective impedance on the interfaces between the bending part and the dielectric waveguides. We envisage that the design would be applicable from the far-infrared to the terahertz frequency range due to the tunable dielectric responses of graphene. 
Waveguide bends are necessary for photonic integration and central to electromagnetic wave communication and manipulation. Conventional waveguide bends usually suffer from low transmission or serious wave-front distortion. Many solutions, such as plasmonic bends ${ }^{1)}$ and photonic crystal bends, ${ }^{2)}$ have been proposed to improve the bending performance of waveguides. In recent years, with the development of metamaterials, arbitrary waveguide bends were proposed under the framework of transformation optics ${ }^{3)}$ and geometric optics. ${ }^{4)}$ A 90-degree bend structure was realized experimentally with gradient index and isotropic metamaterials. ${ }^{5)}$ Meanwhile, Han and coworkers successfully designed adaptive waveguide bends with homogeneous, isotropic materials; ${ }^{6}$ Luo et al. proposed a so-called perfect bending waveguide based on realizable anisotropic epsilon-near-zero metamaterials. ${ }^{7)}$ This waveguide was experimentally verified in the microwave region by Ma et al. ${ }^{8)}$ Harnessing both zero-phase variation and uniform field-distribution properties, zero-index metamaterials with deliberately engineered defects are powerful for tunneling, ${ }^{9,10)}$ total reflection or total transmission. ${ }^{11,12)}$ Recently, anisotropic zero-index metamaterials were also shown to be capable of arbitrarily manipulating the energy flux. ${ }^{13,14)}$

However, existing bends and zero-index metamaterials are universally challenged by their lack of adjustability and are limited by their applicable frequency range. For example, metamaterials made of split-ring resonators ${ }^{8)}$ or I-shaped unit cells ${ }^{5)}$ are sensitive to the wavelength and are difficult to extend to the high-frequency band. Fortunately, the use of graphene may help to resolve these issues because graphene has been shown to have high carrier mobility and feasible tunability ${ }^{15,16)}$ with electric gating or doping in the electromagnetic regime. Graphene has previously been used in field transistors, ${ }^{17)}$ wave modulators, ${ }^{18)}$ polarizers, ${ }^{19)}$ absorbers ${ }^{20)}$ and sensors. ${ }^{21,22)}$ For example, we recently reported an enhanced light-graphene interaction platform by combing graphene with lab-on-a-rod techniques. ${ }^{19)}$ Alternately aligned graphene and metal gratings were designed to achieve Fano-type resonance by exploiting their different responses to electromagnetic waves. ${ }^{22)}$ Furthermore, metamaterials based on graphene/dielectric multilayers have also been studied both theoretically ${ }^{23,24)}$ and experimentally. ${ }^{25,26)}$ The strong coupling between the surface plasmon polaritons in graphene sheets makes graphene a powerful material for controlling wave radiation $^{27)}$ and for designing ultrasensitive modulators. ${ }^{28)}$ Graphene-based metamaterials should also be beneficial in bending electromagnetic waves with proper designs.

In this letter, we propose tunable waveguide bends using graphene-based anisotropic metamaterials (GBAM) made of graphene layers that are alternately stacked between 
dielectric layers. We use effective medium theory to understand the anisotropic dielectric property of GBAM. Nearly perfect bending is achieved by tuning graphene's Fermi level to realize a zero index along the propagating direction. Our structure is applicable for different periods of GBAM and for different bending shapes. Using GBAM, we can arbitrarily guide and bend electromagnetic waves over a large frequency range, properties that are promising for applications in telecommunications and photonics.

A schematic of a two-dimensional (2D) bend is presented in Fig. 1(a). Regions 1 and 3 are dielectric waveguides with relative permittivity, $\varepsilon_{\mathrm{d}}$. Region 2 is our designed GBAM made of multi-concentric layers of periodically altered dielectric and graphene with the radial direction normal to the surfaces of the layers. The GBAM is sandwiched between two metal layers that can serve as parallel electrodes to uniformly bias the chemical potential of graphene. In the far infrared and terahertz regime, these metal walls can be regarded as perfect metal and perfect conducting condition is therefore employed. If the metal boundaries are replaced by a low-index dielectric medium, the functionality of the electrodes will be disabled and wave field will be diffracted and penetrate into the walls of the waveguides so that it is not possible to achieve perfect bending effect. The GBAM between the metal walls can be treated as a uniform medium whose permittivity tensor is expressed as $\left[\varepsilon_{\mathrm{r}}, \varepsilon_{\theta}, \varepsilon_{\mathrm{z}}\right]$ in cylindrical coordinates, with subscripts $\mathrm{r}, \theta$ and $\mathrm{z}$ denoting the radial, azimuthal and $\mathrm{z}$ axes, respectively. The inner and outer radii of the bend are $r_{1}$ and $r_{2}$, respectively. We assume that there is a transverse magnetic (TM) wave incidence from Region 1, i.e., the magnetic field $\vec{H}=\left(0,0, \mathrm{H}_{\mathrm{z}}\right)$ is along the z-axis. No magnetic effect is considered here, and all regions have isotropic permeability, $\mu_{0}$. According to Maxwell's equations, fields in Region 2 obey the following relationship:

$$
\varepsilon_{r} \frac{\partial E_{r}}{\partial t}=\frac{1}{r} \frac{\partial H_{z}}{\partial \theta}, \varepsilon_{\theta} \frac{\partial E_{\theta}}{\partial t}=\frac{\partial H_{z}}{\partial r} .
$$

Thus, we could infer that when $\varepsilon_{\theta}$ is zero, $\mathrm{H}_{\mathrm{z}}$ should be a constant along the radial direction and a TM wave front remains in the bending process. Besides, if the radial component of the permittivity tensor, $\varepsilon_{\mathrm{r}}$, equals $\varepsilon_{\mathrm{d}}$, the wave impedances of the three regions match (i.e., $\sqrt{\mu_{0} / \varepsilon_{r}}=\sqrt{\mu_{0} / \varepsilon_{d}}$ ) and the reflection at the interfaces between different regions vanishes. We performed numerical simulations with COMSOL Multiphysics (a commercial software based on the finite-element method) to validate the bending effect of this anisotropic material. As a proof of concept, we set $\varepsilon_{\theta}=0, \varepsilon_{\mathrm{r}}=\varepsilon_{\mathrm{d}}=2$ and chose the vacuum wavelength $\lambda_{0}=10 \mu \mathrm{m}$, which is about the working wavelength of a $\mathrm{CO}_{2}$ laser. The magnetic field distribution inside such a waveguide bend is shown in Fig. 1(d), where a perfect bending 
effect without any wave front distortion can be seen.

For our proposed GBAM, the dielectric spacers have thickness $t_{d}$ and they also have the same permittivity, $\varepsilon_{\mathrm{d}}$, as the waveguide's materials. In the far-infrared and terahertz regions, where the photon energy is much smaller than graphene's biased Fermi level, $\mathrm{E}_{\mathrm{f}}$, only the intraband electron transition dominates and graphene's surface conductivity can be simplified to $\sigma_{g}=\mathrm{ie}^{2} E_{f} /[\pi \hbar(\hbar \omega+i 2 \Gamma)]$, where e is the electron's charge, $\mathrm{E}_{\mathrm{f}}$ is the Fermi level, $\Gamma$ is the intrinsic loss and $\omega$ is the angular frequency of the incident wave. ${ }^{29)}$ To approximate the GBAM as an effective medium, the graphene is treated as a dielectric layer with very small thickness, $t_{\mathrm{g}}=1 \mathrm{~nm}$, and its permittivity is derived from $\varepsilon_{g}=\varepsilon_{0}+i \sigma_{g} /\left(\omega t_{g}\right)$, where $\varepsilon_{0}$ is the vacuum permittivity. ${ }^{30)}$ When the thickness of the spacer, $t_{d}$, is much smaller than the considered wavelength, the effective permittivity tensor of the GBAM can be determined by the following effective medium theory: ${ }^{31)}$

$$
\varepsilon_{\perp}=\varepsilon_{r}=\frac{\varepsilon_{g} \varepsilon_{d}\left(t_{g}+t_{d}\right)}{t_{g} \varepsilon_{d}+t_{d} \varepsilon_{g}}, \quad \varepsilon_{\|}=\varepsilon_{\theta}=\varepsilon_{z}=\frac{t_{g} \varepsilon_{g}+t_{d} \varepsilon_{d}}{t_{g}+t_{d}} .
$$

From Eq. (2), we can obtain that $\varepsilon_{\mathrm{r}}$ approximately equals $\varepsilon_{\mathrm{d}}$ when the spacer's thickness, $\mathrm{t}_{\mathrm{d}}$, is considerably larger than $\mathrm{t}_{\mathrm{g}}$, meaning that $\varepsilon_{\mathrm{r}}$ will not be affected by graphene's properties and the impedance-matching condition is satisfied. On the other hand, $\varepsilon_{\theta}$ is tunable when the Fermi level or the periodicity of graphene changes. We plot the real (Fig. 1(b)) and imaginary (Fig. 1(c)) parts of $\varepsilon_{\theta}$ versus the $\mathrm{E}_{\mathrm{f}}$ for various $\mathrm{t}_{\mathrm{d}}$. Here, we again set $\varepsilon_{\mathrm{d}}=2$ and $\lambda_{0}=10 \mu \mathrm{m}$. The dashed line in Fig. 1(b) signifies the zero line of the real part of $\varepsilon_{\theta}$. We observe that the larger the period, the higher the Fermi level required for $\operatorname{Real}\left(\varepsilon_{\theta}\right)=0$. We also find that the required Fermi levels are much larger than the photon energy $(\mathrm{E} \approx 0.12$ $\mathrm{eV}$ ), which justifies our calculation of graphene's conductivity by ignoring the interband electron transition. Meanwhile, when $\operatorname{Real}\left(\varepsilon_{\theta}\right)=0$, the corresponding imaginary parts of $\varepsilon_{\theta}$ for different periods are all about 0.02, shown as the dashed line in Fig. 1(c). This means that the metamaterials's dissipation is similar for different combinations of periods and Fermi levels. 
(a)
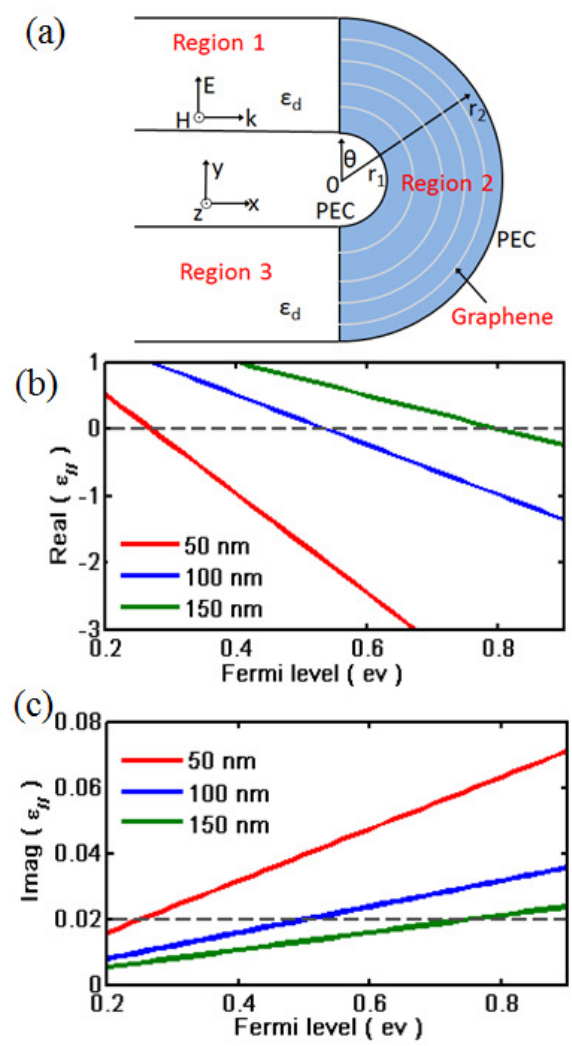

(d)

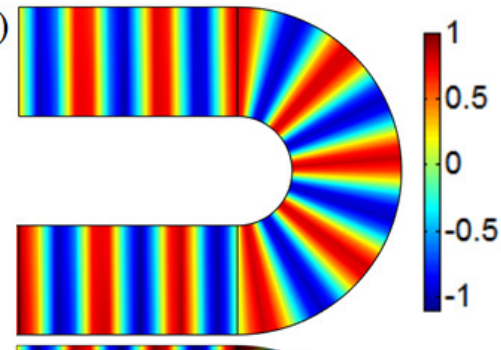

(e)

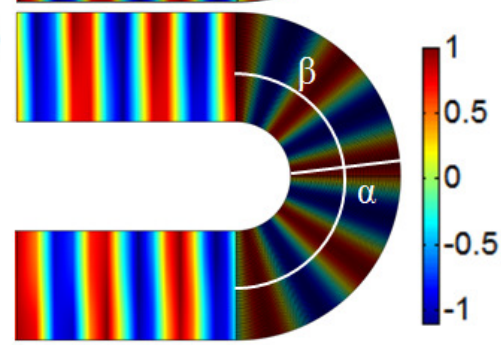

(f)

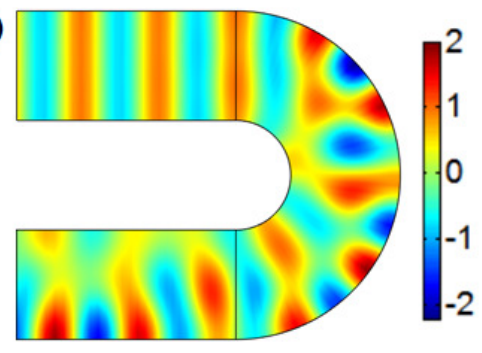

Fig. 1. (a) A schematic diagram of the bend. A transverse magnetic wave launches from Region 1 with magnetic field $\mathrm{H}$ along the z-axis. Regions 1 and 3 are metallic waveguides filled with the dielectric of $\varepsilon_{\mathrm{d}}$. Region 2 is made of GBAM and the gray curves represent graphene layers equally spaced between dielectric layers, $\varepsilon_{d}$, with thickness $t_{d}$ (not proportionate to the real design). (b-c) Real and imaginary parts of GBAM's $\varepsilon_{\|}$for different $t_{d}$ and Fermi levels. Other parameters are $\lambda_{0}=10 \mu \mathrm{m}, \Gamma=0.1 \mathrm{meV}$ and $\varepsilon_{\mathrm{d}}=2$. Black dashed lines $\operatorname{signify} \operatorname{Real}\left(\varepsilon_{\|}\right)=0$ and $\operatorname{Imag}\left(\varepsilon_{\|}\right)=0.02$. (d-f) Magnetic field distributions in a $180^{\circ}$ bending filled with anisotropic dielectric $\left(\varepsilon_{\theta}=0\right.$ and $\left.\varepsilon_{\mathrm{r}}=2\right)$, GBAM and isotropic dielectric $(\varepsilon=2)$, respectively. $\lambda_{0}=10 \mu \mathrm{m}, \mathrm{r}_{1}=5 \mu \mathrm{m}, \mathrm{r}_{2}=\mathrm{r}_{1}+\lambda_{0}$. For GBAM in (e), the period is $100 \mathrm{~nm}$ and the Fermi level is $0.53 \mathrm{ev}$. $\alpha$ and $\beta$ are curves in the radial and azimuthal directions with $\theta=$ $85^{\circ}$ and $\mathrm{r}=10 \mu \mathrm{m}$, respectively.

We further perform numerical simulations to verify the validity of using GBAM for the perfect bending effect. We set the width of the bending waveguide to $r_{2}-r_{1}=\lambda_{0}$ and assume that $r_{1}=5 \mu \mathrm{m}$ is the inner radius of the bend. The dielectric/graphene period in the GBAM is chosen to be $100 \mathrm{~nm}$, and the magnetic field amplitude of the TM incidence is set at $1 \mathrm{~A} / \mathrm{m}$. The outer metallic boundaries of the GBAM can be simply treated as perfect electric boundaries in the simulation considering the metal's relatively low dissipation in the far-infrared and terahertz range. As shown in Fig. 1(e), near-perfect $180^{\circ}$ bending is achieved when $\mathrm{E}_{\mathrm{f}}=0.53 \mathrm{eV}$ at which $\varepsilon_{\theta} \approx 0$. Compared with Fig. 1(d), the shape of the 
wave front is well preserved in the bend, and there is almost no reflection across the interfaces on the GBAM. This result confirms that GBAM behaves exactly like a homogeneous, anisotropic medium with $\varepsilon_{\theta}=0$ and $\varepsilon_{\mathrm{r}}=2$ when the Fermi level of graphene is properly tuned. By contrast, Fig. 1(f) shows the field distribution by the same incident wave but with the GBAM replaced by the isotropic dielectric. Significant wave front distortion is observed.

To better understand the mechanism of the perfect bending effect with GBAM, we select two curves along the radial and azimuthal directions in the bending region, marked as $\alpha$ and $\beta$ in Fig. 1(e), and plot the magnetic field distributions. According to Eq. (1), uniform magnetic field distribution along curve $\alpha$ is expected when $\varepsilon_{\theta}=0$. Figure 2(a) verifies this conclusion by showing that the magnitude of $\mathrm{H}_{\mathrm{Z}}$ is about $0.95 \mathrm{~A} / \mathrm{m}$, together with many sharp peaks. In the vicinity of the graphene sheets, Eq. (1) can be adapted into

$$
\varepsilon_{\theta} \frac{\partial E_{\theta}}{\partial t}+\mathrm{J}_{\theta}=\frac{\partial H_{z}}{\partial r}
$$

where $\mathbf{J}_{\theta}$ is the surface current on graphene induced by the plasmon resonance. As a result, sharp changes in the magnetic field are observed near the surface of the graphene as exhibited in the inset of Fig. 2(a).

The plasmonic effect is also manifested in Fig. 2(b), where the small oscillations are induced by interference between the incident wave and the plasmonic wave of the graphene. We can exactly calculate the wave vector of the surface plasmon polariton of the graphene by

$$
\mathrm{k}_{S P P}=k_{0} \sqrt{\varepsilon_{d}+\left(\frac{2 i \varepsilon_{d}}{\eta_{0} \sigma_{g}}\right)^{2}} .
$$

Here, $\eta_{0}$ is the intrinsic impedance of free space. ${ }^{27)}$ The wavelength of the graphene plasmon is thus $\lambda_{\mathrm{SPP}}=2 \pi / \operatorname{Real}\left(\mathrm{k}_{\mathrm{SPP}}\right) \approx 0.31 \mu \mathrm{m}$, matching the interference pattern in Fig. 2(b) well. Meanwhile, the EM wave suffers propagation loss in the bending process as the amplitude of the magnetic field decreases from 1 to $0.9 \mathrm{~A} / \mathrm{m}$ along the azimuthal direction. Since we can get $\operatorname{Imag}\left(\mathrm{k}_{\mathrm{SPP}}\right) \propto \varepsilon_{\mathrm{d}}$ from Eq. (4), a dielectric with smaller $\varepsilon_{\mathrm{d}}$ is preferred to reduce the bending dissipation.

In Fig. 2(c), we plot in blue curve the Fermi levels (calculated from Eq. (2)) that are needed to obtain $\operatorname{Real}\left(\varepsilon_{\theta}\right)=0$ with different periods. This figure shows that a larger period requires a higher Fermi level. For each period, we also perform numerical simulations by tuning the Fermi level of the graphene layers to achieve the perfect bending effect, i.e., uniform magnetic field distribution along the radial direction in GBAM and no wave front distortion. These results are plotted in Fig. 2(c) (red dots), which indicates excellent 
agreement with the prediction of Eq. (2), validating that effective medium theory is able to describe GBAM.
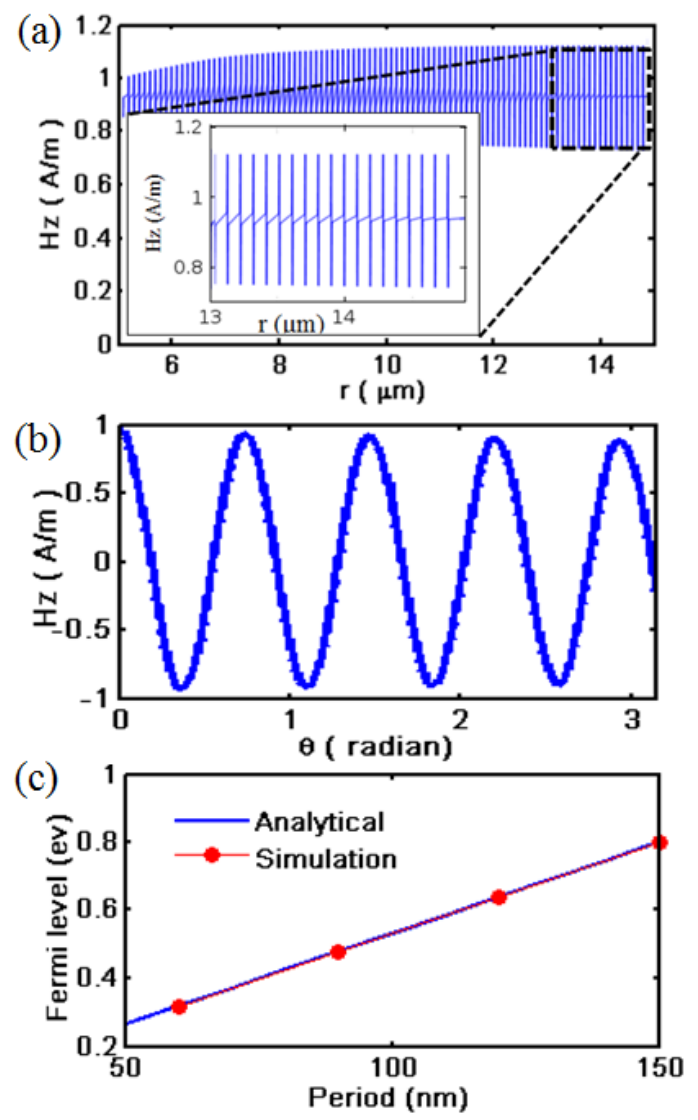

Fig. 2. (a-b) Magnetic field distributions at curves $\alpha$ and $\beta$ marked in Fig. 1(e) respectively. (c) Fermi level required for $\varepsilon_{\theta}=0$. Results of the analytical calculations (blue line) match the numerical simulations (red dots) well.

Here, a perfect bending effect with GBAM is successfully demonstrated, and the validity of effective medium theory in describing the anisotropic properties of the metamaterials is verified. In what follows, we study the tunability of the bend as well as the performance of the bend with different bending curvatures and shapes. First, the adjustability of graphene's permittivity permits the application of our GBAM bending structure within a large frequency regime. We calculate the Fermi level required for $\varepsilon_{\theta}=0$ with different periods and frequencies. As shown in Fig. 3(a), the working frequency of the GBAM bend can be extended from the far-infrared to the terahertz region as long as we change the Fermi level of graphene within a reasonable range. ${ }^{32)}$ For a given structure (period), it is possible to guide the wave perfectly by changing the Fermi level to achieve the required effective medium profile of the GBAM at different frequencies. Theoretically, we could arbitrarily guide the incident wave with GBAM without energy reflection or 
wave front distortion as long as we kept $\varepsilon_{\theta}=0$ by tuning graphene's Fermi level.

The effect of the bend curvature is next studied by changing the inner radius, $r_{1}$. Transmissivity versus $r_{1}$ is plotted in Fig. 3(b). Though the transmissivity slightly decreases when we maintain the waveguide width and reduce $r_{1}$, perfect bending functionality of GBAM persists, as shown in the inset with $r_{1}=2 \mu \mathrm{m}$. Larger curvature results in stronger surface current dissipation on graphene, accounting for the decrease in the transmissivity. In Fig. 3(c), we further simulate $360^{\circ}$ bending when other parameters are the same as those in the case shown in Fig. 1(e). The wave front remains well preserved after transmitting through the bending waveguide.
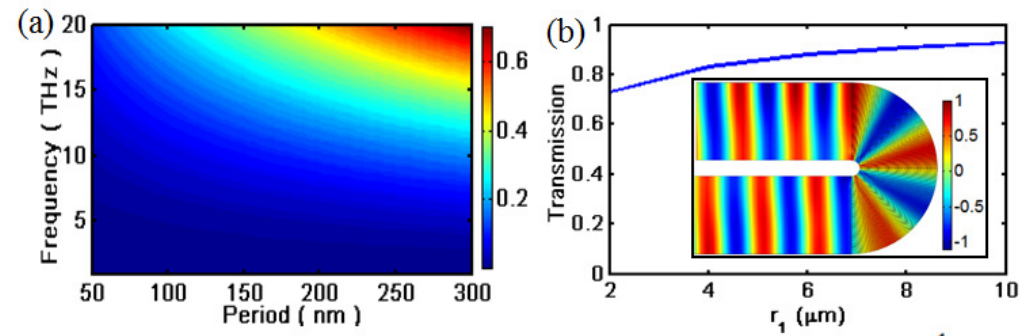

(c)

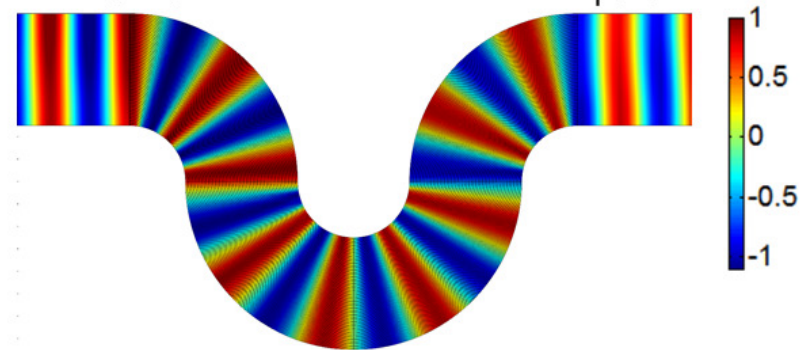

Fig. 3. (a) The Fermi level required for $\varepsilon_{\|}=0$ at different periods and frequencies. The unit of the colorbar is ev. (b) Transmittance of $180^{\circ}$ bending with different $r_{1}$. The inset shows the magnetic field distribution when $\mathrm{r}_{1}=2 \mu \mathrm{m}$. Other parameters are the same as those used in Fig. 1(e). (c) Magnetic field distribution in $360^{\circ}$ bending filled with GBAM. Other parameters are the same as those used in Fig. 1(e).

In conclusion, we proposed perfect bending of TM waves using graphene-based anisotropic metamaterials made of multi-concentric layers of graphene and dielectric. Tuning graphene's Fermi level can give rise to a zero effective index along the propagating direction such that the TM wave front is preserved and the perfect bending effect is achieved. We investigated the magnetic field distributions to validate bending functionality. The exceptional elasticity and flexible tunability of graphene's electric response enables the design of bends with different curvatures, shapes and frequency ranges.

\section{Acknowledgments}

The work described in this paper was supported by King Abdullah University of Science 
and Technology (KAUST), the National Science Foundation of China (NSFC) under contact no. 61225026, and the Program for Changjiang Scholars and Innovative Research Teams at the University under contract IRT13021. 


\section{References}

1) G. Veronis and S. Fan, Appl. Phys. Lett. 87, 131102 (2005).

2) J. C. Chen, I. Kurland, S. Fan, P. R. Villeneuve, and J. D. Joannopoulos, Phys. Rev. Lett. 77, 3790 (1996).

3) W. Jiang, T. Cui, X. Zhou, X. Yang, and Q. Cheng, Phys. Rev. E 78, 066607 (2008).

4) Z. L. Mei and T. J. Cui, J. Appl. Phys. 105, 104913 (2009).

5) Z. L. Mei and T. J. Cui, Opt. Express 17, 18363 (2009).

6) T. Han, C.-W. Qiu, and X. Tang, Opt. Lett. 36, 181-183 (2011).

7) J. Luo, P. Xu, H. Chen, B. Hou, L. Gao, and Y. Lai, Appl. Phys. Lett. 100, 221903 (2012).

8) H. Feng Ma, J. Hui Shi, W. Xiang Jiang, and T. Jun Cui, Appl. Phys. Lett. 101, 253513 (2012).

9) M. Silveirinha and N. Engheta, Phys. Rev. Lett. 97, 157403 (2006).

10) B. Edwards, A. Alù, M. Young, M. Silveirinha, and N. Engheta, Phys. Rev. Lett. 100, 033903 (2008).

11) V. Nguyen, L. Chen, and K. Halterman, Phys. Rev. Lett. 105, 233908 (2010).

12) Y. Wu and J. Li, Appl. Phys. Lett. 102, 183105 (2013).

13) J. Luo, W. Lu, Z. Hang, H. Chen, B. Hou, Y. Lai, and C. T. Chan, Phys. Rev. Lett. 112, 073903 (2014).

14) J. Luo and Y. Lai, Sci. Rep. 4, 5875 (2014).

15) F. Xia, H. Wang, D. Xiao, M. Dubey, and A. Ramasubramaniam, Nat. Photon. 8, 899-907 (2014).

16) L. Ju, B. Geng, J. Horng, C. Girit, M. Martin, Z. Hao, H. A. Bechtel, X. Liang, A. Zettl, Y. R. Shen, and F. Wang, Nat. Nanotech. 6, 630-634 (2011).

17) P. B. Bennett, Z. Pedramrazi, A. Madani, Y.-C. Chen, D. G. de Oteyza, C. Chen, F. R. Fischer, M. F. Crommie, and J. Bokor, Appl. Phys. Lett. 103, 253114 (2013).

18) X. Gan, R.-J. Shiue, Y. Gao, K. F. Mak, X. Yao, L. Li, A. Szep, D. Walker, J. Hone, T. F. Heinz, and D. Englund, Nano Lett. 13, 691-696 (2013).

19) J.-1. Kou, J.-h. Chen, Y. Chen, F. Xu, and Y.-q. Lu, Optica 1, 307-310 (2014).

20) R. Alaee, M. Farhat, C. Rockstuhl, and F. Lederer, Opt. Express 20, 28017-28024 (2012).

21) A. Davoyan, V. Popov, and S. Nikitov, Phys. Rev. Lett. 108, 127401 (2012).

22) Z.-x. Chen, J.-h. Chen, Z.-j. Wu, W. Hu, X.-j. Zhang, and Y.-q. Lu, Appl. Phys. Lett. 104, 161114 (2014).

23) M. A. K. Othman, C. Guclu, and F. Capolino, J. Nanophot. 7, 15 (2013). 
24) A. Andryieuski, A. Lavrinenko, and D. Chigrin, Phys. Rev. B 86, 121108 (2012).

25) H. Yan, X. Li, B. Chandra, G. Tulevski, Y. Wu, M. Freitag, W. Zhu, P. Avouris, and F. Xia, Nat. Nanotech. 7, 330 - 334 (2012).

26) Z. Xu, C. Chen, S. Q. Y. Wu, B. Wang, J. Teng, C. Zhang, and Q. Bao, Micro/Nano Materials, Devices, and Systems, Proc. SPIE 8923, 89230C (2013).

27) B. Wang, X. Zhang, F. García-Vidal, X. Yuan, and J. Teng, Phys. Rev. Lett. 109, 073901 (2012).

28) I. Khromova, A. Andryieuski, and A. Lavrinenko, Laser \& Photon. Rev. 8, 916-923 (2014).

29) A. Vakil and N. Engheta, Science 332, 1291-1294 (2011).

30) A. H. Castro Neto, F. Guinea, N. M. R. Peres, K. S. Novoselov, and A. K. Geim, Rev. of Mod. Phys. 81, 109-162 (2009).

31) C. Argyropoulos, N. M. Estakhri, F. Monticone, and A. Alù, Opt. Express 21, 15037-15047 (2013).

32) P.-Y. Chen and A. Alù, ACS Nano 5, 5855-5863 (2011). 


\section{Figure Captions}

Fig. 1. (a) A schematic diagram of the bend. A transverse magnetic wave launches from Region 1 with magnetic field $\mathrm{H}$ along the z-axis. Regions 1 and 3 are metallic waveguides filled with the dielectric of $\varepsilon_{\mathrm{d}}$. Region 2 is made of GBAM and the gray curves represent graphene layers equally spaced between dielectric layers, $\varepsilon_{d}$, with thickness $t_{d}$ (not proportionate to the real design). (b-c) Real and imaginary parts of GBAM's $\varepsilon_{\|}$for different $\mathrm{t}_{\mathrm{d}}$ and Fermi levels. Other parameters are $\lambda_{0}=10 \mu \mathrm{m}, \Gamma=0.1 \mathrm{meV}$ and $\varepsilon_{\mathrm{d}}=2$. Black dashed lines signify $\operatorname{Real}\left(\varepsilon_{\|}\right)=0$ and $\operatorname{Imag}\left(\varepsilon_{\|}\right)=0.02$. (d-f) Magnetic field distributions in a $180^{\circ}$ bending filled with anisotropic dielectric $\left(\varepsilon_{\theta}=0\right.$ and $\left.\varepsilon_{\mathrm{r}}=2\right)$, GBAM and isotropic dielectric ( $\varepsilon=2$ ), respectively. $\lambda_{0}=10 \mu \mathrm{m}, \mathrm{r}_{1}=5 \mu \mathrm{m}, \mathrm{r}_{2}=\mathrm{r}_{1}+\lambda_{0}$. For GBAM in (e), the period is $100 \mathrm{~nm}$ and the Fermi level is 0.53 ev. $\alpha$ and $\beta$ are curves in the radial and azimuthal directions with $\theta=85^{\circ}$ and $\mathrm{r}=10 \mu \mathrm{m}$, respectively.

Fig. 2. (a-b) Magnetic field distributions at curves $\alpha$ and $\beta$ marked in Fig. 1(e) respectively. (c) Fermi level required for $\varepsilon_{\theta}=0$. Results of the analytical calculations (blue line) match the numerical simulations (red dots) well.

Fig. 3. (a) The Fermi level required for $\varepsilon_{\|}=0$ at different periods and frequencies. The unit of the colorbar is ev. (b) Transmittance of $180^{\circ}$ bending with different $r_{1}$. The inset shows the magnetic field distribution when $\mathrm{r}_{1}=2 \mu \mathrm{m}$. Other parameters are the same as those used in Fig. 1(e). (c) Magnetic field distribution in $360^{\circ}$ bending filled with GBAM. Other parameters are the same as those used in Fig. 1(e). 
(a)
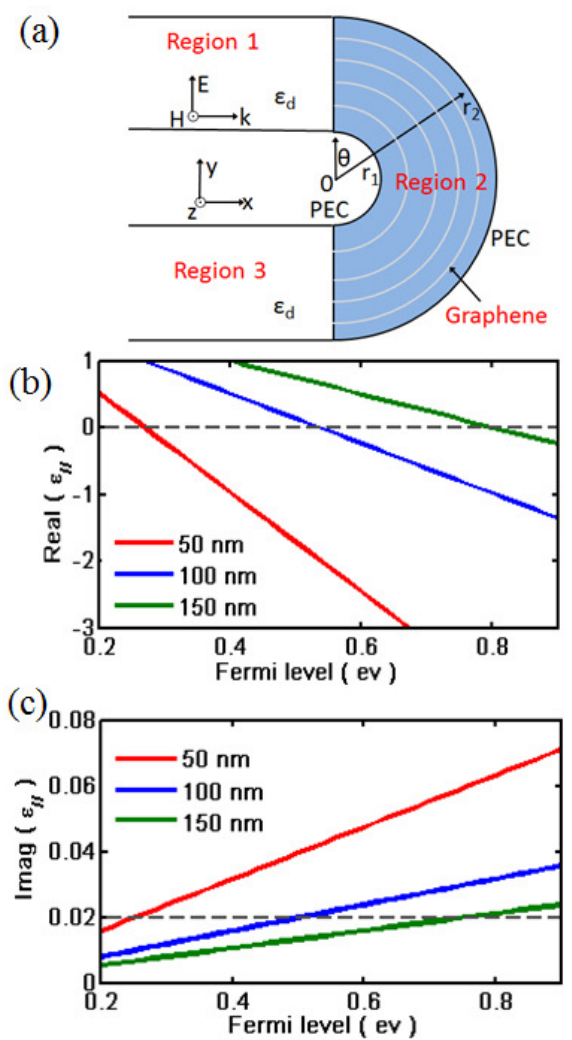

(d)

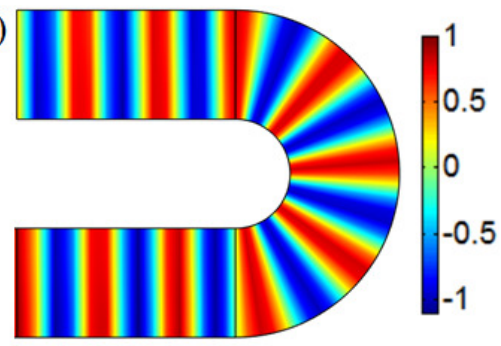

(e)

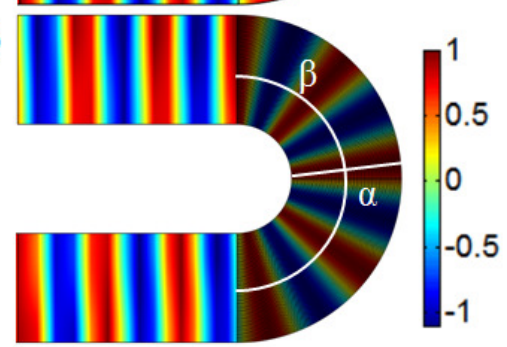

(f)

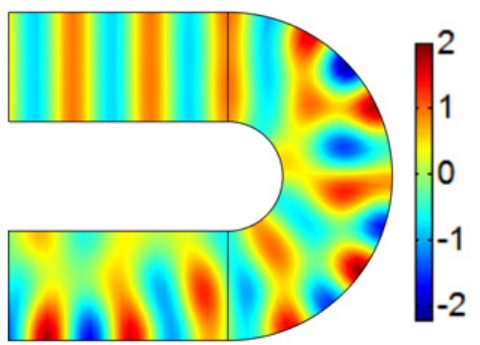

Fig.1. 

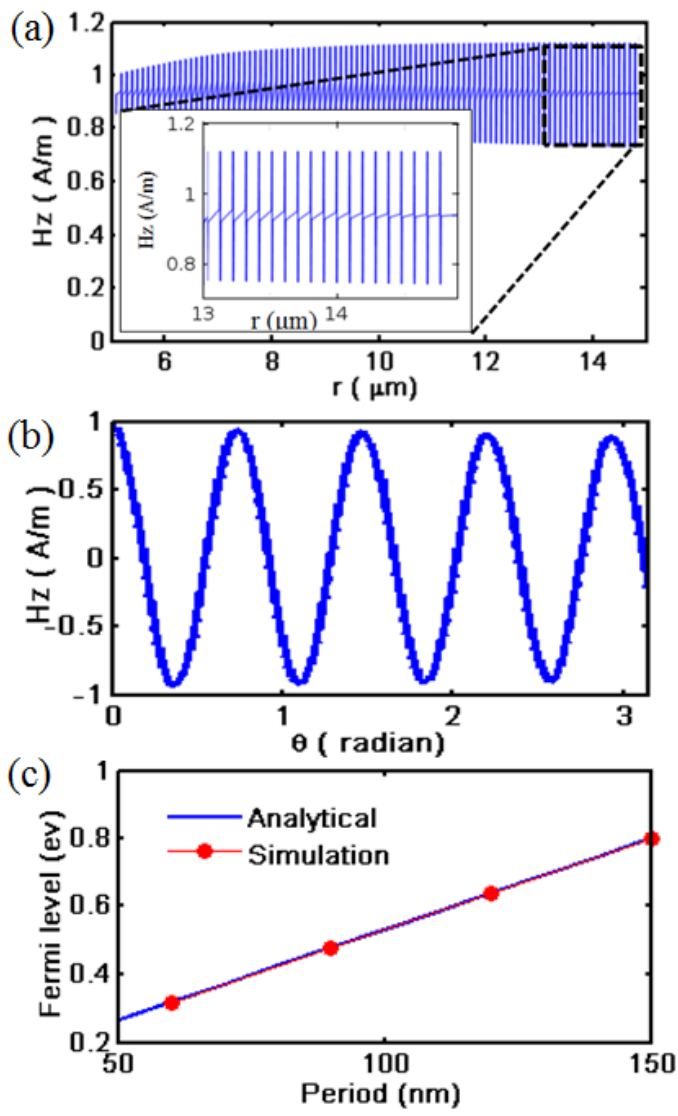

Fig. 2. 

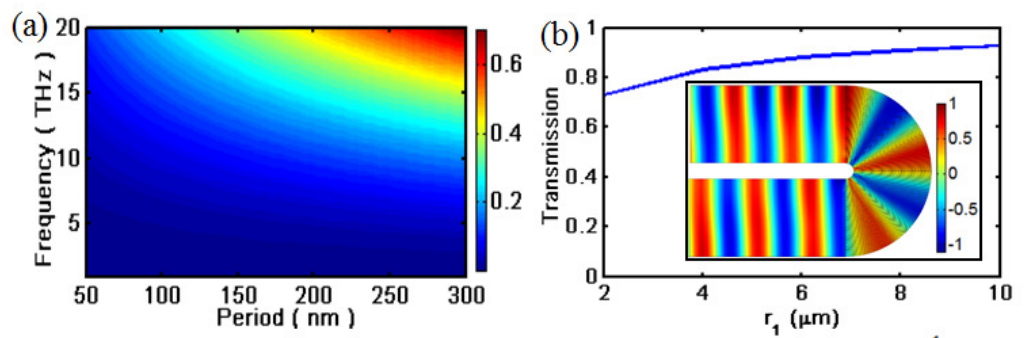

(c)

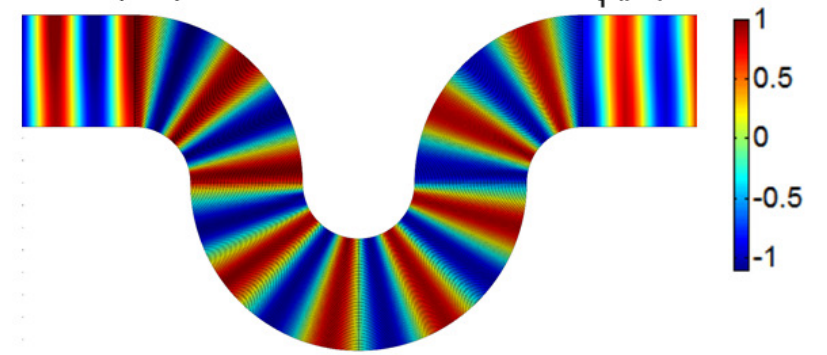

Fig. 3. 